\title{
A Firefly Algorithm for the Mono-Processors Hybrid Flow Shop Problem
}

\author{
Latifa DEKHICI, Khaled BELKADI \\ LAMOSI Laboratory \\ Département d'informatique, \\ Université des Sciences et de la Technnologie d'Oran \\ Mohamed Boudiaf, Algeria
}

\begin{abstract}
Nature-inspired swarm metaheuristics become one of the most powerful methods for optimization. In discrete optimization, the efficiency of an algorithm depends on how it is adapted to the problem. This paper aims to provide a discretization of the Firefly Algorithm (FF) for the scheduling of a specific manufacturing system, which is the mono processors two-stage hybrid flow shop (HFS). This kind of manufacturing system appears in several fields as the operating theatre scheduling problem. Results of proposed discrete firefly algorithm are compared to results of other methods found in the literature. Computational results with different numbers of fireflies and on a standard HFS benchmark of about 55 cases, generating about 1900 instances demonstrates that the proposed discretized metaheuristic reaches the best makespan.
\end{abstract}

Keywords-Firefly algorithm; hybrid flow shop;
metaheuristics; discrete optimization

\section{INTRODUCTION}

Scheduling is an essential decision making task. Short term scheduling consists into allocating resources as machines or persons to perform a set of jobs or tasks to minimize or maximize objective functions. In manufacturing systems, the scheduling objective may be minimization of makespan or Cmax, machine idle time, mean or total flow time and tardiness. Baker [1] addressed different types of scheduling environments in industries. Among them, the hybrid flow shop (HFS) or flexible flow shop problem. This environment, which is the generalization of a flow shop, is made of a set of manufacturing stages. At least one stage may contain several machines. The HFS has a variety of real-industrial applications including ceramic, operating theatre [2] and electronic. HSF scheduling problem was been the interest of many researchers since it was first proposed in [3]. It has been demonstrated to be NP-hard in [4]. Hence, exact methods cannot solve HFS problems. Several methods have been proposed so far to solve HFS scheduling problems. A hybrid heuristic algorithm was addressed to solve the multistage Hybrid Flow Shop problem [5]. A heuristic based on simulated annealing (SA) technique was proposed in [6]. The performance of heuristics in a flow shop scheduling with multiple processors was investigated in [7] where authors studied five heuristics for their performances on makespan and mean flow time criteria in a multiple processors HFS. A generic simulation model for the scheduling problem was detailed in [8] and the task priorities at each stage were established dynamically in order to make easier the performance evaluation of job dispatching priority strategies, concerning the makespan and mean flow time as well as other criteria like average queue length and average resource utilization. Botta-Genoulaz [9] lectured the time windowed multistage version of the problem with identical parallel recourses, when tasks are subject to precedence constraints. She investigated six heuristics to solve minimization of lateness. Hybridation of the Tabu search (TS) with another approach was presented in [10]. Authors considered the manufacturing of concrete blocks in a building industry factory as a hybrid flow shop with the purpose to minimize makespan. With the same purpose, a branch-and-bound algorithm was addressed in [11]. Several heuristics were studied to schedule multiprocessor tasks in the two-stage extension of the same problem in order to minimize makespan as simulating analytics (SA) and TS [12]. In [13] and with recirculation to minimize the weighted number of tardy tasks, authors compare a greedy algorithm with a genetic algorithm (GA) on solving a three-stage HFS scheduling problem. A GA has been applied to solve a more realistic problem with sequence dependent set-up times, numerous manufacturing stages with unrelated parallel machines at each stage and machine eligibility [15]. A hybrid constructive Genetic algorithm was made in [17], advanced GAs with some new machine assignment instructions in [19] and an efficient GA in [22]. Others bio-inspired metaheuristics as an ant colony optimization (ACO) in [16] were studied to solve the HFS scheduling problems. A bat algorithm was adapted in [14]. An effective parallel greedy algorithm (PGA) was addressed in [18] and a particle swarm optimization (PSO) algorithm was studied to solve the flexible flow shop scheduling problems [20]. The PSO was also compared to a bottleneck heuristic to solve the HFS problems [23]. A hybrid artificial neural network (ANN) simulation approach is suggested for solving multi-attribute combinatorial dispatching (MACD) decision problem for scheduling a with multiple processors hybrid flow shop [21].

One can notice that most of papers in HFS literature were addressed to flexible flow shop with multi-processors tasks [55], [56]. In the other hand, some few manuscripts studied the mono processors HSF [24]-[27]. The standard hybrid flow shop or the HSF with mono-processors tasks can be considered as a typical example of scheduling problem and has several applications. One can quote, authors in [2], [28][30] that define a surgeries scheduling problem as a standard hybrid flow shop of two to three stage that are induction stage, 
operating rooms stage and post anesthesia care unit stage. In [31] authors studied an energy aware multi-objective optimization that has a mono-processor tasks flexible flow shop configuration.

In the present paper, the firefly metaheuristic is discretised and adapted to the mono-processor hybrid flow shop problem. For that, in the second section the fireflies in the nature are described. We detailed the firefly algorithm giving a simple pseudo-code. In the third section, the hybrid flow shop problem (HFS) is presented and its notation according to the literature. In the fourth section, an adaptation of the firefly algorithm to the HFS problem and a discretization of fireflies are presented. Also, we describe briefly the particles swarm optimization for the same problem in order to compare it later. In the last section, the results of the discrete firefly algorithm and other implanted algorithms are discussed and compared on a benchmark using improvement rate and average percentage deviation from the lower bound. Finally, we give conclusion and perspectives.

\section{FIREFLy METAHEURISTIC}

In the section below, the firefly algorithm and its principles are described.

\section{A. Inspiration}

Fireflies, as a spice of Lampyridae are small insects with wings talented of producing a cold light flashes in order to attract mates. Their mechanism is supposed to slowly charges until the convinced threshold is obtained, at which they set free the power in the form of light, then the process repeats [32] Firefly algorithm that was first proposed by Yang [33] was inspired by the fireflies mutual attraction and the light decreasing over the distance rather than by the fireflies light flashing phenomenon. Algorithm considers what each firefly observes at the point of its position, when trying to move to a greater light-source.

\section{B. Algorithm}

The Firefly Algorithm is one of the recent nature-inspired metaheuristics developed by the author [33]-[38]. One can find limited articles concerning essentially continuous firefly algorithm [39]-[46]. Continuous firefly algorithm was validated on functions optimization in [36]. A resolution of chaos with firefly can be found in [47]. A hybridisation of the algorithm with genetic was done in [49]. The bi-objective version was proposed in [48]. The first discrete version was adapted to permutation problem in [50] where authors studied flowshop problem using a binary coding of solution and a probability formula for discretization. We can also find other discretization for economic problem such as [32], [51]-[52].

The main firefly algorithm distinctive feature is that it simulates an independent and parallel optimization strategy, where a population or swarm, in each iteration, has generated a number of fireflies. Each one works roughly independently and as a result, the metaheuristic will converge quickly with the fireflies aggregating closely to the optimal solution. The Firefly Algorithm was based on the idealized fireflies behaviour of flashing characteristics. These flashing characteristics were idealized as the three rules below:
1) All fireflies are from the same gender so that one firefly is attracted to another despite their genders.

2) The light intensity or brightness of a firefly is determined by the landscape of the objective function to be optimized.

3) Brightness is proportional to their attractiveness, thus for any two flashing fireflies, the less brighter one will move towards the brighter one. The brightness of both will decrease as their distance increases. If there is not the brightest one than a firefly moves randomly.

According to these three hypotheses, pseudo-code of the Firefly Algorithm (FF) may seem as follows:

Algorithm 1. Pseudo code of the FF Meta-heuristic

\begin{tabular}{|c|c|}
\hline $\begin{array}{l}\text { the } m \\
\mathbf{x}=\left(x_{1},\right. \\
n b)\end{array}$ & 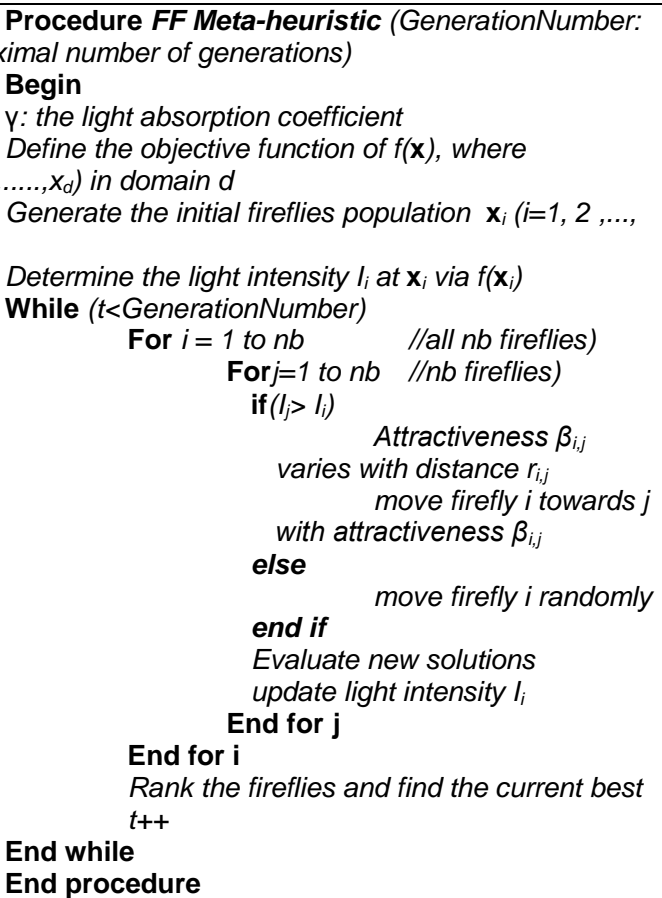 \\
\hline
\end{tabular}

There are four important principles in the Firefly Algorithm:

1) Light Intensity

In the simplest situation for minimum optimization problems, the brightness I of a firefly at a particular location $\mathrm{x}$ can be chosen as $\mathrm{I}(\mathrm{x}) \propto 1 / \mathrm{f}(\mathrm{x})$.

\section{2) Attractiveness}

The principal form of attractiveness function in the firefly optimization can be any monotonically decreasing function such as the generalized form in (1):

$$
\beta_{\mathrm{i}, \mathrm{j}}=\beta_{0}^{*} e^{-\gamma r_{i, j}^{m}}
$$

$r$ is the distance between positions $x_{i}$ and $x_{j}$ of two fireflies $\mathrm{i}$ and $\mathrm{j}$

$\beta_{0}^{*}$ is the attractiveness at $r=0$ and $\gamma$ is a light absorption factor. 


\section{3) Distance}

The distance between two positions $x_{i}$ and $x_{j}$ of two fireflies $\mathrm{i}$ and $\mathrm{j}$ can be the Cartesian distance:

$$
r_{i, j}=\sqrt{\sum_{k=1}^{d}\left(x_{i, k}-x_{j, k}\right)^{2}}
$$

Where $x_{i, k}$ is the $k^{\text {th }}$ part of the $i^{\text {th }}$ firefly.

\section{4) Movement}

The attraction of a firefly $\mathrm{i}$ toward another brighter firefly $\mathrm{j}$, is determined by

$$
x_{i}=\left(1-\beta_{i, j}\right) x_{i}+\beta_{i, j} x_{j}+\alpha(\operatorname{rand}-1 / 2)
$$

where the first and second terms are due to the attraction while the third term is randomization with $\alpha$ being the randomization parameter and "rand" is a random number generator uniformly distributed in $[0,1]$.

\section{Two Stage Hybrid Flow Shop Problem (HFS)}

The Hybrid flow shop scheduling problem will be described in this section.

\section{A. Presentation}

A Hybrid Flow Shop (HFS) also called flexible flow shop is a structure composed of a set of stages, where each stage combines one or more parallel machines. The different tasks visit the stages in the same sequence. On each stage, a job is treated by only one machine. A machine can treat only one job at once. Between each stage, the jobs can wait or not in limited or unlimited buffers.

Moreover, all jobs are assumed to be available at the system entry at date 0 (their release date).

Scheduling in the HFS consists to choose an assignment of the tasks to the range of resources at the various steps and an appropriate sequencing. The purpose is the optimization of one criterion or several performance criteria in case of multipurpose optimization. One can quote the max flow time abbreviated as Fmax, the completion time of the last job on the last stage also called Makespan or Cmax, and due date related purpose.

\section{B. Notation}

Number of stages scheduled is $M$. Number of tasks scheduled is $N$.

$l_{k}$ is the number of machines in stage $k$.

Fig. 1 is an example of a hybrid flow shop with 2 stages and 3 machines on the first stage and 2 machines on the second one. One buffer of infinite capacity is incorporated between stages of the system. The processing time of job $i$ in stage $\mathrm{j}$ if machines are identical is noted $\mathrm{t}_{\mathrm{ij}}$.

Admitting as criterion the Cmax and using Vignier notation [54], the manufacturing shop can be defined by HFS2 $(3,2) \|$ Cmax.

\section{Application}

One can quote as the example of surgeries scheduling in an operating theatre (Fig. 2).

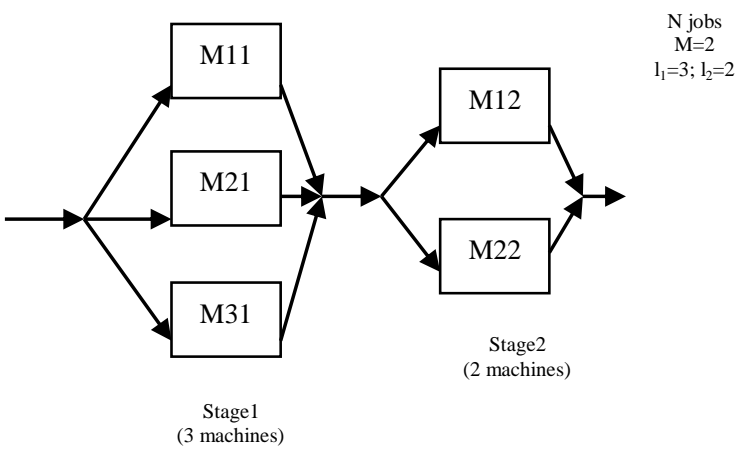

Fig. 1. Representation of HFS2 $\left(l_{1}=3, l_{2}=2\right)||$ Cmax.

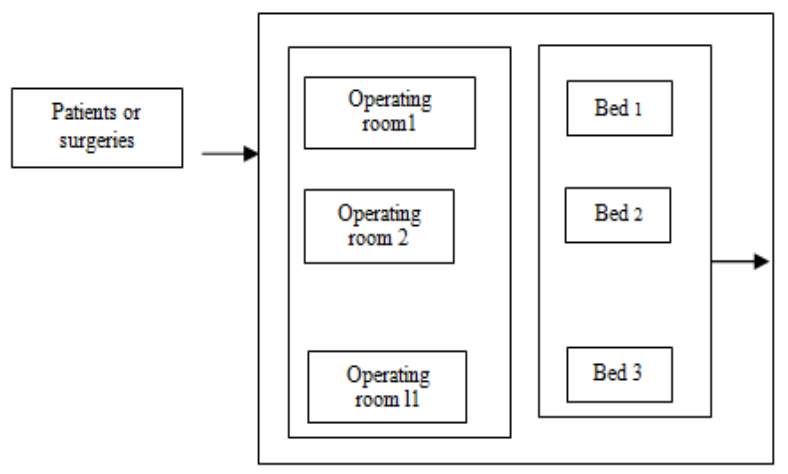

Fig. 2. Example of two stages HFS: operating theatre.

\section{FIREFLY ALGORITHM FOR HSF PROBLEM}

\section{A. Solution Notation}

An integer significant solution coding is adapted to the HSF scheduling. The coding contains two parts: the sequence part $s$ and the assignments part $a$ as one can find in (4).

$$
\begin{array}{r}
x=\left\{s_{i} \mid a_{i k}\right\} 0 \leq i<N, 0 \leq k<M \\
0 \leq s_{i}<N \\
0 \leq a_{i k}<l_{k}
\end{array}
$$

\section{B. Distance}

In this work and since it is a discrete version the distance is replaced by Hamming's distance. The Hamming distance is defined as the number of non-corresponding elements in the sequence or in the assignments (5).

$$
\mathbf{r}_{\mathbf{i}, \mathbf{j}}=\sum_{\mathbf{t}=\mathbf{0}}^{\mathrm{N}-1}\left(\mathbf{s}_{\mathrm{i}, \mathrm{t}} \neq \mathbf{s}_{\mathbf{j}, \mathrm{t}}\right)+\sum_{\mathbf{k}=\mathbf{0}}^{\mathbf{l}_{\mathbf{k}}-\mathbf{1}} \sum_{\mathbf{t}=\mathbf{0}}^{\mathrm{N}-1}\left(\mathbf{a}_{\mathbf{i}, \mathrm{tk}} \neq \mathbf{a}_{\mathbf{j}, \mathrm{tk}}\right)
$$

\section{Random Movement and Other Parameters}

The movement of a firefly $i$ to another $\mathrm{j}$ follows (3) cited earlier. For the alpha coefficient, we choose $\alpha=N$ for the sequence part of the firefly and $\alpha=l_{k} \quad k=1 . . M$ for the assignments part of the firefly.

The random movement of the best firefly uses a neighborhood system based on both sequence permutation and assignment change.

\section{Fireflies Discretization}

When the firefly $i$ moves toward firefly $j$, the position of 
firefly $i$ changes from a binary number $x$ to a real number $x$, (formula 6)

$$
\begin{aligned}
& \mathrm{x}^{\prime}=\left\{\mathrm{s}_{\mathrm{t}}^{\prime} \mid \mathrm{a}_{\mathrm{tk}}^{\prime}\right\} 0 \leq \mathrm{t}<\mathrm{N}, 0 \leq \mathrm{k}<\mathrm{M} \\
& \mathrm{s}_{\mathrm{t}}^{\prime}, \mathrm{a}_{\mathrm{tk}}^{\prime} \in \mathbb{R}
\end{aligned}
$$

To correct sequence, we sort jobs of $s$ per their $s$ ' values. To correct assignments $a$, we adjust $a^{\prime}$ to unsigned integers in the domain $0 . . l_{k}-1$.

\section{E. Particle Swarm Optimization for HFS Problem}

In this sub-section, we describe briefly the particles swarm optimization to compare it with the firefly algorithm.

The Particle Swarm Optimization algorithm (PSO) [53] is initialized with a population of random solutions which is similar in all the evolutionary algorithms. Each individual solution flies in the problem space with a velocity which is adjusted depending on the experiences of the individual and the population

The pseudo code of PSO is given in Algorithm 2.

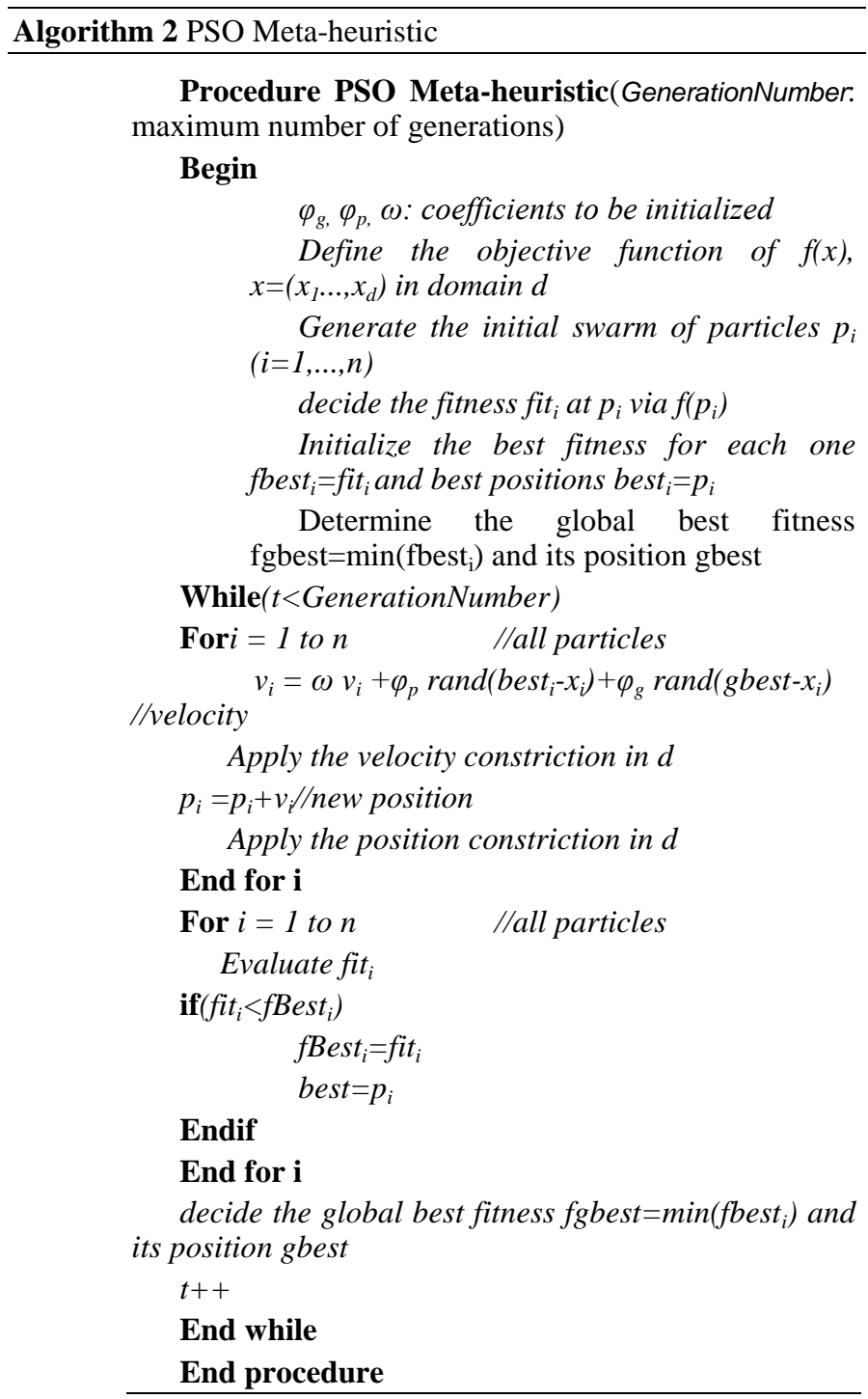

In this study, we retain the principles below:

Fitness. The fitness can be simply the objective function.

Velocity. We keep the equation of velocity $v_{i}$ of a solution $\mathrm{p}_{\mathrm{i}}$ as shown in (7).

$$
\begin{aligned}
& v_{i}= \\
& \omega v_{i}+\varphi_{\rho} \text { rand }()\left(\text { best }_{i}-p_{i}\right)+\varphi_{g} \text { rand }()\left(\text { gbest }_{i}-p_{i}\right)
\end{aligned}
$$

Where $\varphi_{\mathrm{g}}, \varphi_{\mathrm{p}}, \omega$ are coefficients initialized to 1 .

Position. The new position is calculated by (8).

$$
\mathrm{p}_{\mathrm{i}}=\mathrm{p}_{\mathrm{i}}+\mathrm{v}_{\mathrm{i}}
$$

We use the same codification and discretization principles as our Firefly algorithm. We add velocity constriction.

\section{COMPUTATIONAL RESUlTS AND COMPARISON}

\section{A. Hybrid Flow Shop Data}

As a typical sample for experimental comparison, the choice was directed on mono processors two stages hybrid flow shop with related machines that admits that the processing duration of a task do not depend on machine in a stage. A buffer of infinite capacity is integrated between the two stages for each machine. Moreover $\mathrm{N}$ tasks are assumed to be available at the manufacturing shop entrance. The system has $l_{l}$ recourses in the stage one and $l_{2}$ in the second one.

Three categories of instances of two-stage HFS have been randomly generated:

- Category A: These samples were generated in a way similar as in [24]. The number of tasks $n$ is taken from the set $\{20,30,40,50,100\}$. The numbers of resources $(11,12)$ are $(2,2),(2,4)$ and $(4,2)$. The processing durations noted 2:4, 4:2, 4:4 are drawn randomly from a discrete uniform distribution either on $[1,40]$ or $[1$, 20]. These instances characteristics are combined to acquire nine different problem samples for each unchanging N. For each combination, 20 instances were produced which results in 900 instances for the Category A. The category A represents a diversified mix of shop and size setting.

- Category B: It is produced in the same way as in [25]. The number of resources $l_{1}$ and $l_{2}$ were drawn randomly from the discrete uniform distribution on $[2,6]$. The processing durations on stage $\mathrm{j}$ were generated randomly from a discrete uniform distribution on $\left[1,5 * 1_{j}\right](j=1,2)$. The number of task $\mathrm{N}$ was taken equal to $20,30,40,50$ and 100 . For each fixed N, 50 instances were generated. Hence, Category B contains a total number of 250 instances.

- Category C: In this set, the processing durations on both stages were drawn randomly from the discrete uniform distribution on $[1,20]$ [25]. Their 250 instances were generated in a similar way as those of category B. 
For the set $\mathrm{C}$, the workloads are typically unbalanced while for the set B, the workloads at the two stages lean to be well balanced. The population size of particles or fireflies is set to 10 .

The proposed algorithms were implemented in $\mathrm{C}++32$ on an Intel Core 2 Duo 2,53 Giga Hz with aRAM capacity of 6 Giga Byte. If any optimal scheduling was found within the maximum CPU time limit of 15 seconds, the exploration is exited and the best scheduling and it evaluations are output. In order to get good comparison, the choice of the limit criteria lies on the computer setting similarity with these in [28].

\section{B. Comparison Criteria}

In order to compare the proposed discretized algorithms, the measures below can assess their efficiencies:

1) Lower bound LB. Lower Bound indicates the lower makespan can be reached for the HFS scheduling instance. The two stage hybrid flow shop LB retained in this work were presented first time in [24]

$$
L B=\max \left(\frac{\operatorname{spt}\left(l_{1}\right) \sum_{i=1}^{N} t_{i 1}}{l_{1}}, \frac{\operatorname{spt}\left(l_{2}\right) \sum_{i=1}^{N} t_{i 2}}{l_{2}}\right)
$$

Where $\operatorname{spt}\left(1_{1}\right)$ is the minimum sum of completion times, on Stage 2 , of the $l_{1}$ tasks whose processing times on the second stage are the shortest.

2) Average Percentage Deviation (APD). The deviation of a scheduling makespan from the LB is specified in (10):

$$
A P D=\frac{c \max -L B}{L B} \times 100
$$

3) Improvement Rate. Improvement of the final best scheduling from the initial best population is given in (11):

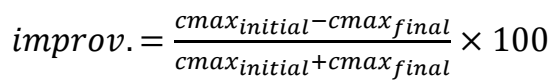

4) Others. We use two other analysis measures:

- TGB that is the average Time to Get the global Best (optimum) when Average Percentage Deviation $=0$.

- NS that is the number of instances for which optimality was proved (Number of Solved). In [28], authors used UnSolved instances (US) factor which is the number of instances not solved.

\section{Results and Discussion}

First, we compare the firefly algorithms with Particle Swarm Optimization. Table I shows Average Deviations, average improvements and Makespan, min and max criteria values. We observe that Firefly algorithm solved to optimally all of instances in $86.67 \%$ of the problems (39 per 45). The most of Firefly Algorithm unsolved instances are with unbalanced workload from classes where there are less resources in the first stage. The problems are easier for $2 \times 2$ and $4 \times 2$ cases.
We try to compare our results (Table II) with those found in:

- [24] using Tabu search.

- [27] using climbing bounded discrepancy search $\left(\mathrm{CDDS}^{\mathrm{L}}\right)$.

- [25] using Branch and Bound method (CPU time limit for the Backward or Forward problems was set equal to $600 \mathrm{~s}$.)

- [28] using Climbing Depth-bounded Discrepancy Search $\left(\mathrm{CDDS}^{2}\right)$ (maximum time limit was $15 \mathrm{~s}$ and the number of instances for each class was 20).

The experiments on the first Category $\mathrm{A}$ as revealed in Table II match the previous conclusion and lend further confirmation. Remarkably, the proposed firefly metaheuristic provides the optimum makespans. It doesn't deviate from lower bound in most of cases and their worst deviation are less than 0.1 while the average APD of all instances for respectively tabu search and $\mathrm{B} \& \mathrm{~B}$ algorithm as examples are 0.51 and 0.28 .

Indeed, we observe from Table III when comparing numbers of solved instances and times to get optimum that FF algorithm yields optimal or very near optimal solutions in most Instances (887 per 900) in 0.0331 second while PSO yields optimal solution in only 499 instances within 75.38 hs and respectively CDDS2 and CCDSL yield optimal solution in 829 and 638 instances.

The worst firefly TGB times can be noticed in the $2 \times 4$ problems but do not exceed 40.63 hs while PSO ones reach 86.29 and $1237 \mathrm{hs}$.

In order to investigate the method on other workload categories, 50 instances were generated for each case either on set B or C. The results of sets B and C for N from 20 to 100 are represented in Table IV. The global efficiency of the discretized firefly algorithm is corroborated by the computational results that were reported on the sets $\mathrm{B}$ and $\mathrm{C}$. Table IV approves that the FF algorithm with only 10 fireflies can give an improvement from $11.74 \%$ to $25.51 \%$ and a deviation from $0.07 \%$ to 0 face to PSO improvement which cannot exceed $20.74 \%$ and PSO deviation that can reach $0.43 \%$.

The most striking result to emerge from Table $\mathrm{V}$ is that $63.2 \%$ of the Set B (balanced instances) and $82.2 \%$ of the Set $\mathrm{C}$ (unbalanced instances) were significantly solved to optimality. Face to only $12.8 \%$ of set B and $36 \%$ of set C solved by PSO. The average firefly algorithm TGB is only 131 hundredth second while it is exceed 166 hundredth second by particles swarm algorithm. And even if the B\&B has more solved instances especially for unbalanced hybrid flow shops, our Firefly Algorithm outperforms it in term of deviation in all of instances. Furthermore, the deviation from the best makespan is approximately null. Overall, our algorithm produced proven optimal solutions. 
TABLE I. SET A, AVERAgE Improvement, APD, CMAX, MIN AND MAX CMAX of DifFerent Methods

\begin{tabular}{|c|c|c|c|c|c|c|c|c|c|c|c|c|}
\hline \multicolumn{3}{|c|}{ Problem } & \multicolumn{2}{|l|}{ Cmax } & \multicolumn{2}{|c|}{ Improvement \% } & \multicolumn{2}{|c|}{ APD\% } & \multicolumn{2}{|l|}{ Minf } & \multicolumn{2}{|l|}{ Maxf } \\
\hline & & & PSO & FF & PSO & FF & PSO & FF & PSO & FF & PSO & FF \\
\hline \multirow{9}{*}{ A100 } & \multirow{3}{*}{$2 \times 2$} & $2: 4$ & 1064.4 & 1064 & 5.22 & 5.24 & \begin{tabular}{|l|l|}
0.04 \\
\end{tabular} & 0 & 1064 & 1064 & 1067 & 1064 \\
\hline & & $4: 2$ & 1005.3 & 1003 & 5.1 & 5.24 & 0.27 & 0 & 1003 & 1003 & 1078 & 1003 \\
\hline & & $4: 4$ & 1005.15 & 999 & 4.79 & 5.1 & 0.64 & 0 & 999 & 999 & 1080 & 999 \\
\hline & \multirow{3}{*}{$2 \times 4$} & $2: 4$ & 591 & 526.95 & 7.46 & 13.11 & 12.36 & 0.02 & 544 & 490 & 642 & 578 \\
\hline & & $4: 2$ & 582.25 & 511.1 & 8.24 & 14.66 & 14.14 & 0.05 & 528 & 456 & 634 & 545 \\
\hline & & $4: 4$ & 585.95 & 512.85 & 7.18 & 13.75 & 14.46 & 0.08 & 533 & 463 & 630 & 544 \\
\hline & \multirow{3}{*}{$4 \times 2$} & $2: 4$ & 988.6 & 987 & 4.86 & 4.94 & 0.17 & 0 & 987 & 987 & 1082 & 987 \\
\hline & & $4: 2$ & 1018.1 & 1018 & 4.6 & 4.62 & 0.04 & 0 & 1018 & 1018 & 1163 & 1018 \\
\hline & & $4: 4$ & 1011.75 & 1012 & 5.83 & 5.84 & 0.01 & 0 & 1012 & 1012 & 1085 & 1012 \\
\hline \multirow{9}{*}{ A20 } & \multirow{3}{*}{$2 \times 2$} & $2: 4$ & 195.45 & 192 & 13.39 & 14.23 & 1.91 & 0 & 192 & 192 & 222 & 192 \\
\hline & & $4: 2$ & 206.6 & 204 & 11.98 & \begin{tabular}{|l|}
12.53 \\
\end{tabular} & 1.15 & 0 & 204 & 204 & 238 & 204 \\
\hline & & $4: 4$ & 200.45 & 197 & 12.05 & 12.88 & 1.84 & 0 & 197 & 197 & 244 & 197 \\
\hline & \multirow{3}{*}{$2 \times 4$} & $2: 4$ & 130.6 & 110 & 17.22 & 25.4 & 19.22 & 0 & 114 & 110 & 151 & 110 \\
\hline & & $4: 2$ & 128.7 & 107 & 16.05 & 24.99 & 21.54 & 0 & 109 & 107 & 147 & 107 \\
\hline & & $4: 4$ & 136.15 & 111 & 17.6 & 27.41 & 23.62 & 0 & 111 & 111 & 159 & 111 \\
\hline & \multirow{3}{*}{$4 \times 2$} & $2: 4$ & 199 & 199 & 9.9 & \begin{tabular}{|l|}
9.9 \\
\end{tabular} & 0 & 0 & \begin{tabular}{|l|}
199 \\
\end{tabular} & 199 & 199 & 199 \\
\hline & & $4: 2$ & 203.75 & 204 & 11.45 & 11.51 & 0.14 & 0 & 204 & 204 & 242 & 204 \\
\hline & & $4: 4$ & 206.75 & 207 & 13.25 & 13.25 & 0 & 0 & 207 & 207 & 261 & 207 \\
\hline \multirow{9}{*}{ A30 } & \multirow{3}{*}{$2 \times 2$} & $2: 4$ & 297.85 & 295 & 11.31 & 11.77 & 0.97 & 0 & 295 & 295 & 321 & 295 \\
\hline & & $4: 2$ & 308.8 & 305 & 10.62 & 11.33 & 1.48 & 0 & 305 & 305 & 367 & 305 \\
\hline & & $4: 4$ & 317.35 & 315 & 7.69 & 8.13 & 0.9 & 0 & 315 & 315 & 371 & 315 \\
\hline & \multirow{3}{*}{$2 \times 4$} & $2: 4$ & \begin{tabular}{|l|}
184.1 \\
\end{tabular} & 161 & 13.77 & 20.35 & 15.09 & 0 & 162 & 161 & 200 & 161 \\
\hline & & $4: 2$ & 182.95 & 161 & 16.86 & 22.8 & 13.39 & 0 & 161 & 161 & 222 & 161 \\
\hline & & $4: 4$ & 186.05 & 160.1 & 15.87 & 23.07 & 16.77 & 0.04 & 154 & 133 & 214 & 182 \\
\hline & \multirow{3}{*}{$4 \times 2$} & $2: 4$ & 294.15 & 294 & \begin{tabular}{|l|}
9.46 \\
\end{tabular} & \begin{tabular}{|l|}
9.47 \\
\end{tabular} & \begin{tabular}{|l|}
0.02 \\
\end{tabular} & 0 & 294 & 294 & 348 & 294 \\
\hline & & $4: 2$ & 295 & 295 & 9.19 & 9.19 & 0 & 0 & 295 & 295 & 347 & 295 \\
\hline & & $4: 4$ & 302.9 & 303 & 10.77 & 10.77 & 0 & 0 & 303 & 303 & 357 & 303 \\
\hline \multirow{9}{*}{ A40 } & & $2: 4$ & 399.75 & 399 & 10.27 & 10.44 & 0.37 & 0 & 399 & 399 & 467 & 399 \\
\hline & $2 \times 2$ & $4: 2$ & 403 & 402 & 8.97 & 9.17 & 0.4 & 0 & 402 & 402 & 456 & 402 \\
\hline & & $4: 4$ & 417.8 & 415 & 7.05 & 7.36 & 0.64 & 0 & 415 & 415 & 472 & 415 \\
\hline & & $2: 4$ & 251.45 & \begin{tabular}{|l|}
210.9 \\
\end{tabular} & 12.46 & 21.02 & \begin{tabular}{|l|}
19.65 \\
\end{tabular} & \begin{tabular}{|l|}
0.03 \\
\end{tabular} & 234 & 185 & 275 & 234 \\
\hline & $2 \times 4$ & $4: 2$ & 238.6 & 210 & 13.72 & 19.83 & 13.77 & 0 & 215 & 210 & 262 & 210 \\
\hline & & $4: 4$ & 244.7 & 217 & 13.1 & 19.05 & 13.45 & 0 & 217 & 217 & 276 & 217 \\
\hline & & $2: 4$ & 405.2 & 405 & 6.36 & 6.39 & 0.07 & 0 & 405 & 405 & 478 & 405 \\
\hline & $4 \times 2$ & $4: 2$ & 409 & 409 & 9.71 & 9.71 & 0 & 0 & 409 & 409 & 470 & 409 \\
\hline & & $4: 4$ & 407.2 & 407 & 7.21 & 7.21 & 0 & 0 & 407 & 407 & 476 & 407 \\
\hline & & $2: 4$ & 514.6 & 509 & 7.04 & 7.58 & 1.14 & 0 & 509 & 509 & 593 & 509 \\
\hline & $2 \times 2$ & $4: 2$ & \begin{tabular}{|l|}
498.05 \\
\end{tabular} & 493 & 7.39 & 7.89 & 1.09 & 0 & 493 & 493 & 552 & 493 \\
\hline & & $4: 4$ & 524.4 & 522 & 7.27 & 7.56 & 0.6 & 0 & 522 & 522 & 593 & 522 \\
\hline & & $2: 4$ & 297.9 & 257.5 & 14.8 & 21.83 & 16.09 & 0.1 & 263 & 230 & 339 & 287 \\
\hline A50 & $2 \times 4$ & $4: 2$ & 303.35 & 266 & 12.6 & 19.09 & 14.54 & 0 & 274 & 266 & 338 & 266 \\
\hline & & $4: 4$ & 302 & 259 & 11.72 & 19.17 & 16.86 & 0 & 262 & 259 & 345 & 259 \\
\hline & & $2: 4$ & 497.05 & 496 & 7.44 & 7.51 & 0.15 & 0 & 496 & 496 & 557 & 496 \\
\hline & $4 \times 2$ & $4: 2$ & 505.15 & 505 & 7.48 & 7.49 & 0.02 & 0 & 505 & 505 & 609 & 505 \\
\hline & & $4: 4$ & 493.15 & 493 & 11 & 11.03 & 0.07 & 0 & 493 & 493 & 523 & 493 \\
\hline Avg. & & & 420.92 & 407.32 & 10.16 & 12.68 & 5.76 & 0.01 & 409.53 & 402.38 & 470.04 & 411.56 \\
\hline
\end{tabular}


TABLE II. Set A, Comparison of Average Deviation of Different Methods*

\begin{tabular}{|c|c|c|c|c|c|c|c|c|}
\hline \multirow{2}{*}{ Problen } & & & \multicolumn{6}{|c|}{ APD\% } \\
\hline & & & \multirow{2}{*}{$\begin{array}{c}\text { PSO } \\
0.04\end{array}$} & \multirow{2}{*}{$\begin{array}{l}\mathbf{F F} \\
0.00\end{array}$} & \multirow{2}{*}{$\begin{array}{l}\text { B\&B [25] } \\
0.00\end{array}$} & \multirow{2}{*}{$\begin{array}{l}\text { TS [24] } \\
0.54\end{array}$} & \multirow{2}{*}{$\begin{array}{l}\text { CCDS2 [28] } \\
0.05\end{array}$} & \multirow{2}{*}{$\begin{array}{l}\text { CCDSL [27] } \\
0.23\end{array}$} \\
\hline \multirow{9}{*}{ A100 } & \multirow{3}{*}{$2 \times 2$} & $2: 4$ & & & & & & \\
\hline & & $4: 2$ & 0.27 & 0.00 & 0.00 & 0.26 & 0.00 & 0.09 \\
\hline & & $4: 4$ & 0.64 & 0.00 & 0.00 & $\mathrm{Na}$ & 0.01 & 0.16 \\
\hline & \multirow{3}{*}{$2 \times 4$} & $2: 4$ & 12.36 & 0.02 & 0.00 & 0.19 & 0.06 & 0.3 \\
\hline & & $4: 2$ & 14.14 & 0.05 & 0.00 & 0.07 & 0.00 & 0.09 \\
\hline & & $4: 4$ & 14.46 & 0.08 & 0.00 & 0.11 & 0.00 & 0.15 \\
\hline & \multirow{3}{*}{$4 \times 2$} & $2: 4$ & 0.17 & 0.00 & 0.00 & 0.02 & 0.00 & 0.09 \\
\hline & & $4: 2$ & 0.04 & 0.00 & 0.00 & 0.18 & 0.03 & 0.35 \\
\hline & & $4: 4$ & 0.01 & 0.00 & 0.00 & 0.01 & 0.01 & 0.09 \\
\hline \multirow{9}{*}{ A20 } & \multirow{3}{*}{$2 \times 2$} & $2: 4$ & 1.91 & 0.00 & 0.00 & NA & 0.05 & 0.16 \\
\hline & & $4: 2$ & 1.15 & 0.00 & 0.00 & NA & 0.03 & 0.08 \\
\hline & & $4: 4$ & 1.84 & 0.00 & 0.88 & NA & 0.39 & 0.48 \\
\hline & \multirow{3}{*}{$2 \times 4$} & $2: 4$ & 19.22 & 0.00 & 1.71 & 2.9 & 0.95 & 5.79 \\
\hline & & $4: 2$ & 21.54 & 0.00 & 0.00 & 0.56 & 0.03 & 0.09 \\
\hline & & $4: 4$ & 23.62 & 0.00 & 0.00 & 0.92 & 0.00 & 0.07 \\
\hline & \multirow{3}{*}{$4 \times 2$} & $2: 4$ & 0.00 & 0.00 & 0.00 & 0.35 & 0.00 & 0.12 \\
\hline & & $4: 2$ & 0.14 & 0.00 & 6.31 & 1.22 & 0.75 & 5.70 \\
\hline & & $4: 4$ & 0.00 & 0.00 & 0.00 & 0.13 & 0.05 & 0.23 \\
\hline \multirow{9}{*}{ A30 } & \multirow{3}{*}{$2 \times 2$} & $2: 4$ & 0.97 & 0.00 & 0.00 & NA & 0.02 & 1.61 \\
\hline & & $4: 2$ & 1.48 & 0.00 & 0.00 & NA & 0.00 & 0.16 \\
\hline & & $4: 4$ & 0.9 & 0.00 & 0.00 & $\mathrm{NA}$ & 0.1 & 0.63 \\
\hline & \multirow{3}{*}{$2 \times 4$} & $2: 4$ & 15.09 & 0.00 & 1.68 & 1.43 & 0.92 & 5.61 \\
\hline & & $4: 2$ & 13.39 & 0.00 & 0.00 & 0.27 & 0.00 & 0.12 \\
\hline & & $4: 4$ & 16.77 & 0.04 & 0.00 & 0.57 & 0.07 & 0.08 \\
\hline & \multirow{3}{*}{$4 \times 2$} & $2: 4$ & 0.02 & 0.00 & 0.00 & 0.06 & 0.00 & 0.12 \\
\hline & & $4: 2$ & 0.00 & 0.00 & 6.36 & 1.46 & 0.96 & 5.69 \\
\hline & & $4: 4$ & 0.00 & 0.00 & 0.00 & 0.05 & 0.02 & 0.05 \\
\hline \multirow{9}{*}{ A40 } & & $2: 4$ & 0.37 & 0.00 & 0.00 & NA & 0.00 & 0.04 \\
\hline & $2 \times 2$ & $4: 2$ & 0.4 & 0.00 & 0.00 & NA & 0.00 & 0.06 \\
\hline & & $4: 4$ & 0.64 & 0.00 & 0.00 & NA & 0.08 & 0.26 \\
\hline & & $2: 4$ & 19.65 & 0.03 & 0.00 & 0.96 & 0.21 & 0.97 \\
\hline & $2 \times 4$ & $4: 2$ & 13.77 & 0.00 & 0.00 & 0.34 & 0 & 0.53 \\
\hline & & $4: 4$ & 13.45 & 0.00 & 0.00 & 0.5 & 0.02 & 0.14 \\
\hline & & $2: 4$ & 0.07 & 0.00 & 0.00 & 0.12 & 0.00 & 0.56 \\
\hline & $4 \times 2$ & $4: 2$ & 0.00 & 0.00 & 2.14 & 0.89 & 0.28 & 1.01 \\
\hline & & $4: 4$ & 0.00 & 0.00 & 0.00 & 0.12 & 0.01 & 0.17 \\
\hline & & $2: 4$ & 1.14 & 0.00 & 0.00 & NA & 0.00 & 0.07 \\
\hline & $2 \times 2$ & $4: 2$ & 1.09 & 0.00 & 0.00 & NA & 0.00 & 0.56 \\
\hline & & $4: 4$ & 0.6 & 0.00 & 0.00 & NA & 0.00 & 0.28 \\
\hline & & $2: 4$ & 16.09 & 0.1 & 0.00 & 0.54 & 0.15 & 0.70 \\
\hline A50 & $2 \times 4$ & $4: 2$ & 14.54 & 0.00 & 0.00 & 0.26 & 0.00 & 0.43 \\
\hline & & $4: 4$ & 16.86 & 0.00 & 0.00 & $\mathrm{NA}$ & 0.00 & 015 \\
\hline & & $2: 4$ & 0.15 & 0.00 & 0.00 & 0.02 & 0.00 & 0.11 \\
\hline & $4 \times 2$ & $4: 2$ & 0.02 & 0.00 & 0.00 & 0.04 & 0.37 & 0.69 \\
\hline & & $4: 4$ & 0.07 & 0.00 & 0.00 & NA & 0.02 & 0.05 \\
\hline Avg. & & & 5.76 & 0.01 & 0.28 & 0.51 & 0.13 & 1.36 \\
\hline
\end{tabular}


TABLE III. SET A, COMPARISON OF The Average TGB AND THE NumBer OF SOlved InSTANCES

\begin{tabular}{|c|c|c|c|c|c|c|c|c|}
\hline \multirow{2}{*}{\multicolumn{3}{|c|}{ Problem }} & \multicolumn{2}{|l|}{ TGB } & \multicolumn{4}{|l|}{ NS } \\
\hline & & & PSO & FF & PSO & $\overline{F F}$ & CDDS2 [27] & CDDSL [28] \\
\hline \multirow{9}{*}{ A100 } & \multirow{3}{*}{$2 \times 2$} & $2: 4$ & 3.33 & 1.75 & 15 & 20 & 19 & 16 \\
\hline & & $4: 2$ & 86.29 & 2.85 & 14 & 20 & 20 & 17 \\
\hline & & $4: 4$ & 21.47 & 1.2 & 15 & 20 & 20 & 11 \\
\hline & \multirow{3}{*}{$2 \times 4$} & $2: 4$ & 519 & 16.68 & 1 & 19 & 18 & 14 \\
\hline & & $4: 2$ & - & 7.47 & 0 & 17 & 20 & 18 \\
\hline & & $4: 4$ & - & 11.33 & 0 & 15 & 20 & 19 \\
\hline & \multirow{3}{*}{$4 \times 2$} & $2: 4$ & 47.95 & 0.85 & 19 & 20 & 20 & 15 \\
\hline & & $4: 2$ & 0.72 & 0.6 & 18 & 20 & 18 & 13 \\
\hline & & $4: 4$ & 3.24 & 0.2 & 17 & 20 & 18 & 11 \\
\hline \multirow{9}{*}{ A20 } & \multirow{3}{*}{$2 \times 2$} & $2: 4$ & 0 & 0 & 14 & 20 & 18 & 17 \\
\hline & & $4: 2$ & 85.82 & 0 & 11 & 20 & 19 & 15 \\
\hline & & $4: 4$ & 0.45 & 0 & 11 & 20 & 15 & 9 \\
\hline & \multirow{3}{*}{$2 \times 4$} & $2: 4$ & 1 & 0.05 & 2 & 20 & 12 & 20 \\
\hline & & $4: 2$ & - & 3.65 & 0 & 20 & 20 & 17 \\
\hline & & $4: 4$ & - & 35.4 & 0 & 20 & 20 & 13 \\
\hline & \multirow{3}{*}{$4 \times 2$} & $2: 4$ & 0.5 & 0.25 & 20 & 20 & 20 & 16 \\
\hline & & $4: 2$ & 0 & 0 & 19 & 20 & 15 & 7 \\
\hline & & $4: 4$ & 0.4 & 0.05 & 20 & 20 & 20 & 17 \\
\hline \multirow{9}{*}{ A30 } & \multirow{3}{*}{$2 \times 2$} & $2: 4$ & 0.2 & 0.05 & 15 & 20 & 19 & 13 \\
\hline & & $4: 2$ & 84 & 0 & 12 & 20 & 20 & 16 \\
\hline & & $4: 4$ & 0.77 & 0 & 13 & 20 & 16 & 8 \\
\hline & \multirow{3}{*}{$2 \times 4$} & $2: 4$ & 11 & 0.05 & 2 & 20 & 14 & 3 \\
\hline & & $4: 2$ & - & 0.85 & 0 & 20 & 20 & 17 \\
\hline & & $4: 4$ & - & 0.32 & 0 & 19 & 20 & 12 \\
\hline & \multirow{3}{*}{$4 \times 2$} & $2: 4$ & 0.47 & 0.25 & 19 & 20 & 20 & 17 \\
\hline & & $4: 2$ & 0.4 & 0 & 20 & 20 & 13 & 9 \\
\hline & & $4: 4$ & 9.35 & 0 & 20 & 20 & 19 & 15 \\
\hline \multirow{9}{*}{ A40 } & \multirow{3}{*}{$2 \times 2$} & $2: 4$ & 0.44 & 0.65 & 16 & 20 & 20 & 18 \\
\hline & & $4: 2$ & 0.14 & 0 & 14 & 20 & 20 & 18 \\
\hline & & $4: 4$ & 1.75 & 0.3 & 16 & 20 & 17 & 11 \\
\hline & \multirow{3}{*}{$2 \times 4$} & $2: 4$ & - & 40.63 & 0 & 19 & 16 & 12 \\
\hline & & $4: 2$ & 1237 & 2.2 & 1 & 20 & 20 & 17 \\
\hline & & $4: 4$ & 415 & 11.65 & 2 & 20 & 19 & 13 \\
\hline & \multirow{3}{*}{$4 \times 2$} & $2: 4$ & 2.47 & 0.25 & 19 & 20 & 20 & 16 \\
\hline & & $4: 2$ & 0.3 & 0.2 & 20 & 20 & 17 & 11 \\
\hline & & $4: 4$ & 3.4 & 0.3 & 20 & 20 & 19 & 14 \\
\hline \multirow{9}{*}{ A50 } & & $2: 4$ & 1.31 & 0.9 & 13 & 20 & 20 & 17 \\
\hline & $2 \times 2$ & $4: 2$ & 0.17 & 2.3 & 12 & 20 & 20 & 17 \\
\hline & & $4: 4$ & 44.21 & 1.55 & 14 & 20 & 20 & 14 \\
\hline & & $2: 4$ & - & 1 & 0 & 18 & 18 & 14 \\
\hline & $2 \times 4$ & $4: 2$ & - & 1.7 & 0 & 20 & 20 & 18 \\
\hline & & $4: 4$ & - & 1.25 & 0 & 20 & 20 & 12 \\
\hline & & $2: 4$ & 47.68 & 0.05 & 19 & 20 & 20 & 16 \\
\hline & $4 \times 2$ & $4: 2$ & 1.39 & 0.05 & 18 & 20 & 12 & 10 \\
\hline & & $4: 4$ & 6.72 & 0.05 & 18 & 20 & 18 & 15 \\
\hline & & & 75.38 & \begin{tabular}{|l|l|}
3.31 \\
\end{tabular} & 499 & 887 & 829 & 638 \\
\hline & & & AVG. & & SUM & & & \\
\hline
\end{tabular}

Table IV. Set B and C. Average Criteria of Different Methods with 10 Particles

\begin{tabular}{|c|c|c|c|c|c|c|c|c|c|c|}
\hline \multirow{2}{*}{ Problem } & \multicolumn{2}{|l|}{ Cmax } & \multicolumn{2}{|c|}{ Improvement\% } & \multicolumn{2}{|c|}{ APD\% } & \multicolumn{2}{|l|}{ TGB } & \multicolumn{2}{|l|}{ NS } \\
\hline & PSO & FF & PSO & FF & PSO & FF & PSO & FF & PSO & FF \\
\hline b20 & 60.66 & 54.62 & 20.74 & 25.51 & 0.21 & 0.00 & 84.67 & 130.53 & 12 & 38 \\
\hline b30 & 89.30 & 81.56 & 18.05 & 22.33 & 0.13 & 0.00 & 201.11 & 174.83 & 9 & 42 \\
\hline b40 & 123.62 & 108.60 & 15.85 & 21.99 & 0.31 & 0.07 & 388.14 & 194.90 & 7 & 29 \\
\hline b50 & 148.82 & 134.82 & 15.02 & 19.66 & 0.43 & 0.00 & 290.75 & 146.21 & 4 & 24 \\
\hline b100 & 301.68 & 266.82 & 8.40 & 14.37 & 0.30 & 0.02 & 0.00 & 285.44 & 0 & 25 \\
\hline c20 & 85.16 & 81.00 & 17.74 & 20.88 & 0.00 & 0.00 & 58.73 & 87.70 & 26 & 47 \\
\hline c30 & 110.76 & 105.22 & 18.52 & 21.35 & 0.01 & 0.00 & 130.82 & 73.58 & 17 & 36 \\
\hline c40 & 167.22 & 159.56 & 14.09 & 16.72 & 0.01 & 0.00 & 177.67 & 35.45 & 18 & 44 \\
\hline c50 & 219.18 & 210.68 & 10.37 & 12.72 & 0.13 & 0.00 & 109.52 & 98.36 & 21 & 45 \\
\hline \multirow[t]{3}{*}{ c100 } & 388.14 & 356.64 & 7.04 & 11.74 & 0.20 & 0.01 & 222.25 & 83.60 & 8 & 35 \\
\hline & 169.45 & 155.95 & 14.58 & 18.73 & 0.17 & 0.01 & 166.37 & 131.06 & 122 & 365 \\
\hline & \multicolumn{4}{|l|}{ Avg. } & & & & & \multicolumn{2}{|l|}{ Sum } \\
\hline
\end{tabular}


TABLE V. SET B AND C. COMPARISON OF CRITERIA BetweEn DifFERENT Methods

\begin{tabular}{|c|c|c|c|c|c|c|c|c|}
\hline Problem & \multicolumn{2}{|l|}{ TGB } & \multicolumn{3}{|l|}{ NS } & \multicolumn{3}{|l|}{ APD } \\
\hline SET N & PSO & FF & PSO & FF & B\&B [28] & PSO & FF & B\&B [28] \\
\hline b20 & 84.67 & 130.53 & 12 & 38 & 35 & 0.21 & 0.00 & 3.99 \\
\hline b30 & 201.11 & 174.83 & 9 & 42 & 34 & 0.13 & 0.00 & 3.26 \\
\hline b40 & 388.14 & 194.90 & 7 & 29 & 38 & 0.31 & 0.07 & 4.56 \\
\hline b50 & 290.75 & 146.21 & 4 & 24 & 37 & 0.43 & 0.00 & 2.95 \\
\hline b100 & 0.00 & 285.44 & 0 & 25 & 44 & 0.30 & 0.02 & 1.69 \\
\hline c20 & 58.73 & 87.70 & 26 & 47 & 46 & 0.00 & 0.00 & 3.78 \\
\hline c30 & 130.82 & 73.58 & 17 & 36 & 49 & 0.01 & 0.00 & 1.37 \\
\hline c40 & 177.67 & 35.45 & 18 & 44 & 49 & 0.01 & 0.00 & 0.95 \\
\hline c50 & 109.52 & 98.36 & 21 & 45 & 48 & 0.13 & 0.00 & 3.93 \\
\hline \multirow[t]{3}{*}{ c100 } & 222.25 & 83.60 & 8 & 35 & 49 & 0.20 & 0.01 & 0.52 \\
\hline & 166.366 & 131.06 & 122 & 365 & 429 & 0.173 & 0.01 & 2.7 \\
\hline & \multicolumn{2}{|l|}{ Avg. } & \multicolumn{3}{|l|}{ Sum. } & \multicolumn{3}{|l|}{ Avg. } \\
\hline
\end{tabular}

To test performance of the FF algorithm regarding to its CPU time to get the optimum, we schematize in Fig. 3 the average TGB. The Firefly Algorithm needs less time than PSO algorithm to reach the optimum.

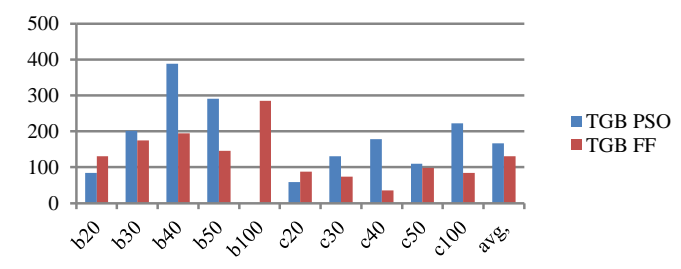

Fig. 3. Set B and C. Comparison of TGB between PSO and FF.

\section{CONCLUSION}

The purpose of this work was the discretization of a Firefly Algorithm to resolve the two-stage mono-processors hybrid flow shop scheduling. This algorithm is a nature-inspired metaheuristic for continuous optimization and the most of the articles found in the literature used it in its continuous version. In this paper, a discretization of the algorithm is given. A meaningful solution encoding of sequencing and assignment is kept. We held Hamming's distance between fireflies to find the more attractive taking that it is more significant for discrete values.

In order to compare the efficiency of the discretized firefly algorithm a particle swarm algorithm was coded with the same principles. Moreover, a standard benchmark was used with 55 scheduling HFS samples with either unbalanced and balanced workloads and heterogeneous settings. A good choice of comparison criteria applied was made as the average percentage deviation from the lower bound.

The discretization and the good choice of attraction parameter permitted to our Firefly Algorithm to catch the best amelioration rate on minimizing makespan criteria in a reasonable execution time.

In conclusion, the Firefly Algorithm was more appropriate to exploit the HSF search space by improving individuals scheduling and simultaneously obtaining the most attractive one.

Additional investigation required to refine the work described in this paper. We are working on others variants of the manufacturing systems such as constrained hybrid flow shops without buffer minimizing blocking duration.

\section{REFERENCES}

[1] Baker. K.R. (1974): Introduction to Sequencing and Scheduling. Wiley. New York.

[2] Dekhici. L., Belkadi.K.: Operating theatre scheduling under constraints. J. Applied Sci.. 2010. 10: 1380-1388.

[3] Arthanary and Ramamurthy, 1971.An extension of two machines sequencing problem. Journal of Operational Research. Vol. 41: 641-648.

[4] Gupta, 1988. Two stage hybrid flowshop scheduling problem. Journal of Operational Research Society. Vol. 39(4): 359-364.

[5] Portmann. M.C., Vignier. A. Dardilhac. D. and Dezalay. D. 1998: Branch and bound crossed with GA to solve hybrid flowshops. European Journal of Operational Research..107: 389-400

[6] Riane, F. (1998). Scheduling hybrid flow shops: Algorithms and application. Thesis at Catholic University of Mons, Belgium.

[7] Brah and Loo, (1999). Heuristics for scheduling in a flow shop with multiple processors. European Journal of Operational Research.Vol.11(3):113-122.

[8] N Grangeon, A Tanguy, N Tchernev. Generic simulation model for hybrid flow-shop. Computers \& industrial engineering, (1999) - Elsevier

[9] Botta-Genoulaz, V., 2000. Hybrid flow shop scheduling with precedence constraints and time lags to minimize maximum lateness. Int. J. Prod. Econ., 64: 101-111.

[10] J. Grabowski, J. Pempera. Sequencing of jobs in some production system. European Journal of Operational Research.Vol. 125(3): 535-550 (2000)

[11] O. Moursli, Y. Pochet.A branch-and-bound algorithm for the hybrid flow shop . International Journal of Production Economics, Vol. 64, Issues 1-3, 1 (2000):113-125

[12] A Janiak, E Kozan, M Lichtenstein, C Oğuz.Metaheuristic approaches to the hybrid flow shop scheduling problem with a cost-related criterion. International journal of production economics 105 (2), 407-424

[13] Bertel S., J-C Billaut; "A genetic algorithm for an industrial multiprocessor flow-shop scheduling problem with recirculation". EJOR.159(3) :651-662, (2004)

[14] MK Marichelvam, T Prabaharam.(2013).A bat algorithm for realistic hybrid flowshop schedulihng problems to minimize makespan and mean flow time. ICTACT Journal on Soft Computing 3 (1), 428-433

[15] R Ruiz, C Maroto(2006),A genetic algorithm for hybrid flowshops with sequence dependent setup times and machine eligibility.European Journal of Operational Research 169 (3), 781-800

[16] Alaykiran, K., Engin, O., Doyen, A., (2007). Using ant colony optimization to solvehybrid flow shop scheduling problems. International Journal of AdvancedManufacturing Technology 35, 541550

[17] Shiau D-F, Cheng S-C, and Huang Y. (2008). Proportionate flexible flow shop scheduling via a hybrid constructive genetic algorithm. Expert Syst. Appl. 34, 2 (February 2008), 1133-1143.

[18] C. Kahraman , O. Engin , İ. Kaya , R. E. Öztürk, Multiprocessor task scheduling in multistage hybrid flow-shops: A parallel greedy algorithm approach, Applied Soft Computing, v.10 n.4, p.1293-1300, September, (2010). 
[19] Urlings, T.; Ruiz, R.; Stutzle, T. Shifting representation search for hybrid flexible flowline problems. European Journal of Operational Research. v207. 1086-1095. (2010)

[20] Manas Ranjan Singh, S. S. Mahapatra A swarm optimization approach for flexible flow shop scheduling with multiprocessor tasks. The International Journal of Advanced Manufacturing Technology.(2011), Vol. 62(1-4): 267-277

[21] A Azadeh, A Naghavi, M Moghaddam,(2011), A hybrid artificial neural network: computer simulation approach for scheduling a flow shop with multiple processors. International Journal of Industrial and Systems Engineering 7 (1), 66-89

[22] O Engin, G Ceran, MK YilmazAn efficient genetic algorithm for hybrid flow shop scheduling with multiprocessor task problems. Applied Soft Computing, (2011). Elsevier

[23] Ching-Jong Liao, Evi Tjandradjaja, Tsui-Ping Chung,(2012). An approach using particle swarm optimization and bottleneck heuristic to solve hybrid flow shop scheduling problem, Applied Soft Computing,Vol 12(6):1755-1764.

[24] Haouari. M.. , M'Hallah. R. (1997). Heuristic algorithms for the twostage hybrid flowshop problem. Operations Research Letters. 21. 43-53.

[25] Haouari. M.. Hidri. L.. \& Gharbi. A. (2006). Optimal scheduling of a two hybrid flow shop. Mathematical Methods of Operations Research. 64. 107-124

[26] Ben Hamida. A.. Huguet. M.-J. Lopez. P.. \& Haouari. M. (2007). Climbing Discrepancy Search for solving the hybrid Flow shop. European Journal of Industrial Engineering. 1(2). 223-243.

[27] Ben Hamida A. Haouari M. Huguet M-J. Lopez P.(2011) Solving twostage hybrid flow shop using climbing depth-bounded discrepancy search. Computers \& Industrial Engineering.Vol. 60(2):320-327.

[28] Dekhici, L.; Belkadi, K. Bi-objective Operating Theater scheduling case of the paediatric hospital of Oran; Logistics and Operations Management (GOL), 2014 International Conference on; 2014, Page(s): $181-187$.

[29] A. Guinet, S. Chaabane Operating theatre planning International Journal of Production Economics, Vol. 85(1) (2003) : 69-81

[30] A. Jebali, A. B. Hadj Alouane, P. LadeT. Operating rooms scheduling . International Journal of Production Economics, Vol. 99 (1-2), 2006: 5262

[31] Ji-hong Yan, Fen-yang Zhang, Xin Li, Zi-mo Wang, Wei Wang . Modeling and Multiobjective Optimization for Energy-Aware Hybrid Flow Shop Scheduling. In Proc. of 4th International Asia Conference on Industrial Engineering and Management Innovation (IEMI2013) 2014, : 741-751

[32] Durkota K.: Implementation of a discrete firefly algorithm for the QAP problem within the sage framework. BSc thesis. Czech Technical University. 2011

[33] Yang X.S: Nature-Inspired Metaheuristic Algorithms. LuniverPress.UK. 2008.

[34] Yang X.S.: Firefly Algorithms for Multimodal Optimisation. Stochastic Algorithms: Foundations and Applications. SAGA 2009. Lecture Notes in Computer Science. Springer-Verlag. Berlin.2009.5792:169-178.

[35] Yang X.S. : Firefly algorithm. stochastic test functions and design optimization.International Journal of Bio-Inspired Computation. 2010. 2(2):78-84.

[36] Yang X.S.: Firefly algorithm. Lévy flights and global optimization. in: Research and Development in Intelligent Systems XXVI (Eds M. Bramer. R. Ellis. M. Petridis). Springer London. 2010. 209-218.

[37] Yang X.S. Review of Metaheuristics and Generalized Evolutionary Walk Algorithm. Int. J. Bio-Inspired Computation. 2011.3(2):77-84.

[38] Yang XS. Multiobjective firefly algorithm for continuous optimization. Engineering with Computers. April 2013. 29(2): 175-184
[39] Lukasik. S. and Zak. S: Firefly Algorithm for Continuous Constrained Optimization Tasks.Lecture Notes in Computer Science. 2009. 5796/2009: 97-106

[40] Horng M-H. and T-W. Jiang: The Codebook Design of Image Vector Quantization Based on the Firefly Algorithm. Lecture Notes in Computer Science. 2010. 6423/2010: 438-447.

[41] Fizazi H.. Beghoura M.A.: Segmentation des Images Satellitaires par l'Algorithme Firefly flou. journées du laboratoire d'informatique d'Oran JDLIO.Oran.2011.

[42] Aungkulanon. P. Chai-ead N. and Luangpaiboon P: Simulated Manufacturing Process Improvement via Particle Swarm optimisation and Firefly Algorithms. In Proc. International Multi Conference of Engineers and Computer Scientists IMECS'11.Hong Kong. 2011. 11231128.

[43] Anirban Chatterjee, Gautam Kumar Mahanti and Gourab Ghatak, Synthesis of satellite footprint patterns from rectangular planar array antenna by using swarm-based optimization algorithms, Int. J. Satell. Commun. Network. 2014; 32:25-47

[44] B. Maher, A. Albrecht, M. Loomes, X. S. Yang, K. Steinhofel, A firefly-inspired method for protein structure prediction in lattice models, Biomolucules, vol. 4, no. 1, pp. 56-75 (2014).

[45] Tao Xiong, Yukun Bao, Zhongyi Hu: Multiple-output support vector regression with a firefly algorithm for interval-valued stock price index forecasting. Knowl.-Based Syst. 55: 87-100 (2014).

[46] Yudong, Zhang; Lenan, Wu; Shuihua, Wang (2013). "Solving TwoDimensional HP model by Firefly Algorithm and Simplified Energy Function". Mathematical Problems in Engineering 2013.

[47] Gandomi A.H,X.-S. Yang, S. Talatahari, A.H. Alavi (2013) Firefly algorithm with chaos. Communications in Nonlinear Science and Numerical Simulation. 18(1):89-98.

[48] Gandomi A.H. Yang X-S.. Alavi A.H. (2011).: Mixed variable structural optimization using Firefly Algorithm. Comput Struct. 89 : 2325-2336.

[49] Shaik Farook P, Sangameswara R. (2013) Evolutionary Hybrid GeneticFirefly Algorithm for Global Optimization. IJCEM, International Journal of Computational Engineering \& Management.16( 3) ISSN : 2230-7893

[50] Sayadi M. K.. R. Ramezanian. N. Ghaffari-Nasab: A Discrete Firefly Meta-heuristic with Local Search for Make span Minimisation in Permutation Flow Shop Scheduling Problems". International Journal of Industrial Engineering Computations. 2010. 1:1-10.

[51] Basu B. and MahantiG. K.: Firefly and artificial bees colony algorithm for synthesis of scanned and broadside linear array antenna. Progress In Electromagnetics Research B. 2011. 32:169-190.

[52] Jati G. K. and SuyantoS.: Evolutionary discrete firefly algorithm for travelling salesman problem. ICAIS2011. Lecture Notes in Artificial Intelligence (LNAI 6943).2011.393-403.

[53] Kennedy. J.. Eberhart. R.C.: Particle swarm optimization. In: Proc. of IEEE International Conference on Neural Networks. Piscataway. NJ.1995.1942-1948.

[54] Vignier A. Billaut. J.C. and Proust. C.: Scheduling problems type hybrid flow-shop: State of the art. RAIRO.Recherche opérationnelle. 1999. 33(2): 117-183.

[55] Marichelvam, M. K., Prabaharan, T., \& Yang, X. S. (2014). A discrete firefly algorithm for the multi-objective hybrid flowshop scheduling problems. IEEE transactions on evolutionary computation, 18(2), 301305.

[56] Marichelvam, M. K., Prabaharan, T., \& Geetha, M. (2015). Firefly Algorithm for Flow Shop Optimization. In Recent Advances in Swarm Intelligence and Evolutionary Computation (pp. 225-243). Springer International Publishing. 